\title{
Parity-violating electron scattering and strangeness form factors of the nucleon
}

\section{Luigi Capozza*}

Institut für Kernphysik,

Johannes Gutenberg-Univeristät, Mainz

E-mail: capozza@kph.uni-mainz.de

In this contribution a brief review of the measurement of the nucleon strangeness vector form factors is given. These observables and the principle of the access to them through parity-violating electron scattering are recalled. A survey of data published by the various experiments is compiled. An account on the needed experimental techniques and more in detail of the A4 experiment at the MAMI facility is presented. Finally the latest results of the A4 collaboration are reported.

6th International Workshop on Chiral Dynamics, CD09

July 6-10, 2009

Bern, Switzerland

${ }^{*}$ Speaker. 


\section{Introduction}

An unsolved puzzle of the low energy dynamics of the strong interaction concerns the relation between the short distance degrees of freedom (DOF) of QCD and the long distance hadron structure. A direct link between these two regimes is given in principle by matrix elements of quark operators evaluated over the wave function of hadrons. One possibility for accessing the quark contribution to non-perturbative hadron properties like form factors, anomalous magnetic moments or charge radii is given by flavour decomposition, i.e. trying to separate the contributions of single quark flavours.

Looking at the valence sector though, e.g. at the $u$ and $d$ flavours in the nucleons, one might be sensitive to some sort of effective DOF, i.e. constituent quarks, which could have little to do with the QCD-quarks, as we learn from chiral quark models [1]. The sea-quarks instead can maybe be identified with the DOF of the QCD lagrangian, motivating the interest for strange quark effects on the nucleon structure.

This contribution concentrates on the strange quark vector current operator $\bar{s} \gamma^{\mu} s$, which contributes to the vector coupling of the nucleons and thus for instance to the electromagnetic form factors. It is accessible in neutral current experiments, as proposed in [2]. In sec. 2 the definition of flavour form factors and their separation through neutral current observables are recalled. The experimental technique of parity-violating electron scattering is reviewed in sec. 3, together with the data which are available so far. A more detailed description of the A4 experiment at Mainz is given in sec. 4 and the latest $\mathrm{A} 4$ results are reported in sec. 5 .

\section{Flavour form factors and the weak neutral current}

The coupling of the electromagnetic field to the hadrons is described in QCD by the elementary vector coupling to the quark fields through the vector current operators $\bar{f} \gamma^{\mu} f$ (quark field operators are denoted by their flavour $f$ ). Thus the electromagnetic current of the nucleon should emerge as a combination of matrix elements of such quark current operators $\left\langle\mathbf{N}\left|\bar{f} \gamma^{\mu} f\right| \mathrm{N}\right\rangle$ and at low momentum transfers only the light flavours $f=u, d, s$ need to be considered. On the other end the the same nucleon electromagnetic current can be parametrised for the elastic scattering amplitude under the assumption of Lorentz invariance, parity invariance and current conservation via the elastic electromagnetic form factors $F_{1}$ and $F_{2}$ :

$$
\left\langle J_{\gamma}^{\mu}\right\rangle=\sum_{f=u, d, s}-i e Q_{f}\left\langle\mathrm{~N}\left|\bar{f} \gamma^{\mu} f\right| \mathrm{N}\right\rangle=-i e \overline{\mathrm{N}}\left(p^{\prime}\right)\left[\gamma^{\mu} F_{1}\left(q^{2}\right)+i \frac{\sigma^{\mu v} q_{v}}{2 M} F_{2}\left(q^{2}\right)\right] \mathrm{N}(p) .
$$

This equation offers a link between fundamental QCD matrix elements and measurable quantities like the form factors. Furthermore one can define flavour form factors by means of (2.1):

$$
\left\langle\mathrm{N}\left|\bar{f} \gamma^{\mu} f\right| \mathrm{N}\right\rangle \equiv \overline{\mathrm{N}}\left(p^{\prime}\right)\left[\gamma^{\mu} F_{1}^{f}\left(q^{2}\right)+i \frac{\sigma^{\mu v} q_{v}}{2 M} F_{2}^{f}\left(q^{2}\right)\right] \mathrm{N}(p),
$$

which are related to the nucleon form factors by

$$
F_{1,2}\left(q^{2}\right)=\sum_{f=u, d . s} Q_{f} F_{1,2}^{f}\left(q^{2}\right) \quad G_{E, M}\left(q^{2}\right)=\sum_{f=u, d . s} Q_{f} G_{E, M}^{f}\left(q^{2}\right),
$$


where the Sachs form factors $G_{E, M}^{(f)}$ are defined as $G_{E}^{(f)}=F_{1}^{(f)}-\tau F_{2}^{(f)}$ and $G_{M}^{(f)}=F_{1}^{(f)}+F_{2}^{(f)}$ ( $\tau=-q^{2} / 4 M_{N}^{2}$ ) and $Q_{f}$ is the electric charge of the $f$-quark.

There are six flavour form factors for each nucleon whereas only two electromagnetic form factors can be measured. In order to reduce the number of unknowns one can make use of isospin symmetry. This implies that the $u$-quark in the proton "behaves" like the $d$-quark in the neutron and vice versa, while the $s$-quark matrix elements are the same in both nucleons. Formally one can write:

$$
G_{E, M}^{u, \mathrm{p}}=G_{E, M}^{d, \mathrm{n}} \equiv G_{E, M}^{u}, \quad G_{E, M}^{d, \mathrm{p}}=G_{E, M}^{u, \mathrm{n}} \equiv G_{E, M}^{d}, \quad G_{E, M}^{s, \mathrm{p}}=G_{E, M}^{s, \mathrm{n}} \equiv G_{E, M}^{s} .
$$

The effects of isospin symmetry violation have been estimated in [3] to be about one order of magnitude smaller than the typical experimental errors of the involved observables and can be safely neglected. Thanks to isospin symmetry both nucleons can be described by a total of six independent flavour form factors $G_{E, M}^{u, d, s}$. Still there are only four measurable form factors $G_{E, M}^{\mathrm{p}, \mathrm{n}}$.

The missing constraints for isolating the single flavour contributions to the nucleon vector coupling can be found in weak neutral current observables, as pointed out by Kaplan and Manohar [2]. The nucleon coupling to the $Z^{0}$ boson can be written as

$$
\left\langle J_{\mathrm{Z}}^{\mu}\right\rangle=\frac{-i e}{2 \sin 2 \theta_{W}} \overline{\mathrm{N}}\left(p^{\prime}\right)\left[\gamma^{\mu} \tilde{F}_{1}\left(q^{2}\right)+i \frac{\sigma^{\mu v} q_{v}}{2 M} \tilde{F}_{2}\left(q^{2}\right)+\gamma^{\mu} \gamma_{5} G_{A}\left(q^{2}\right)\right] \mathrm{N}(p) \equiv\left\langle V_{Z}^{\mu}\right\rangle+\left\langle A_{Z}^{\mu}\right\rangle,
$$

where $\left\langle V_{Z}^{\mu}\right\rangle$ and $\left\langle A_{Z}^{\mu}\right\rangle$ are the vector and the axial part of the current, respectively. For each nucleon three new, in principle measurable form factors $\left(\tilde{F}_{1}, \tilde{F}_{2}\right.$ and $\left.G_{A}\right)$ appear in (2.5). In analogy to (2.1) the vector part of $\left\langle J_{\mathrm{Z}}^{\mu}\right\rangle$ can be written as

$$
\left\langle V_{\mathrm{Z}}^{\mu}\right\rangle=\sum_{f=u, d, s} \frac{-i e}{2 \sin 2 \theta_{W}} Q_{f}^{w}\left\langle\mathrm{~N}\left|\bar{f} \gamma^{\mu} f\right| \mathrm{N}\right\rangle
$$

where the matrix elements $\left\langle\mathrm{N}\left|\bar{f} \gamma^{\mu} f\right| \mathrm{N}\right\rangle$ are the same of those in (2.1), because of the universality of the quark vector current operators, and $Q_{f}^{w}=2 I_{3}-4 Q_{f} \sin ^{2} \theta_{W}$ is the standard model weak charge of the quark. As a result the weak vector form factors of the nucleon can be written as a linear combination of the same flavour form factor appearing in (2.3):

$$
\tilde{F}_{1,2}\left(q^{2}\right)=\sum_{f=u, d . s} Q_{f}^{w} F_{1,2}^{f}\left(q^{2}\right) \quad \tilde{G}_{E, M}\left(q^{2}\right)=\sum_{f=u, d . s} Q_{f}^{w} G_{E, M}^{f}\left(q^{2}\right) .
$$

An extensive review of this formalism can be found in [4].

\section{Parity-violating electron scattering}

The weak vector form factors can be accessed experimentally through electroweak elastic scattering. The electron-proton elastic scattering cross section at the tree level in the standard model contains the contributions

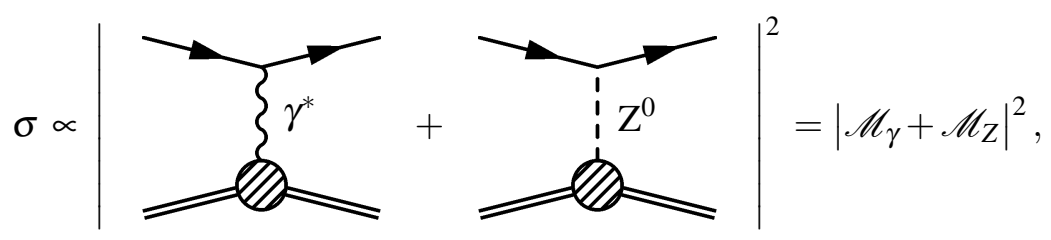


where

$$
\mathscr{M}_{\gamma}=-\frac{j_{\gamma} \cdot\left\langle J_{\gamma}\right\rangle}{q^{2}}, \quad \quad \mathscr{M}_{Z}=-\frac{j_{Z} \cdot\left\langle J_{Z}\right\rangle}{M_{Z}^{2}}+\mathscr{O}\left(q^{2} / M_{Z}^{2}\right) .
$$

In the last equations $j_{\gamma}$ and $j_{Z}$ represent the electron electromagnetic and neutral weak current respectively. The last one possesses a vector and an axial part $j_{Z}^{\mu}=v^{\mu}+a^{\mu}$. The parity nonconservation of the weak interaction can be exploited in order to distinguish phenomenologically between the two amplitudes in (3.1). In the scattering of longitudinally polarised electrons the cross section $\sigma_{R, L}$ has a parity-violating asymmetry $A^{\mathrm{PV}}=\left(\sigma_{R}-\sigma_{L}\right) /\left(\sigma_{R}+\sigma_{L}\right)$ which is sensitive to the interference between the $\gamma$ and the $Z^{0}$ exchange diagram and reads

$$
A^{\mathrm{PV}}=-\frac{q^{2}}{M_{Z}^{2}} \frac{\left(a_{\mu}^{R}-a_{\mu}^{L}\right)\left\langle V_{Z}^{\mu}\right\rangle+\left(v_{\mu}^{R}-v_{\mu}^{L}\right)\left\langle A_{Z}^{\mu}\right\rangle}{j_{\gamma} \cdot\left\langle J_{\gamma}\right\rangle}+\mathscr{O}\left(\left(q^{2} / M_{Z}^{2}\right)^{2}\right),
$$

At the energies of interest here one has typically $\left|q^{2}\right|<1(\mathrm{GeV} / c)^{2}$ and thus higher orders in $q^{2} / M_{Z}^{2}$ need not to be considered.

Considering (2.3) and (2.7) the asymmetry (3.2) depends on the six independent vector flavour form factors and on the axial nucleon form factor $G_{A}$. It can be written in terms of the four nucleon electromagnetic and the strangeness vector form factors $G_{E, M}^{S}$ :

$$
\begin{aligned}
A^{\mathrm{PV}} & =\overbrace{A_{\mathrm{V}}+A_{\mathrm{A}}}^{A_{0}}+A_{\mathrm{S}}: \\
A_{\mathrm{V}} & =-a \rho_{e q}^{\prime}\left[\left(1-4 \hat{\kappa}_{e q}^{\prime} \hat{s}_{Z}^{2}\right)-\frac{\varepsilon G_{E}^{\mathrm{p}} G_{E}^{\mathrm{n}}+\tau G_{M}^{\mathrm{p}} G_{M}^{\mathrm{n}}}{\varepsilon\left(G_{E}^{\mathrm{p}}\right)^{2}+\tau\left(G_{M}^{\mathrm{p}}\right)^{2}}\right] \\
A_{\mathrm{A}} & =a \frac{\left(1-4 \hat{s}_{Z}^{2}\right) \sqrt{1-\varepsilon^{2}} \sqrt{\tau(1+\tau)} G_{M}^{\mathrm{p}} G_{A}^{\mathrm{p}}}{\varepsilon\left(G_{E}^{\mathrm{p}}\right)^{2}+\tau\left(G_{M}^{\mathrm{p}}\right)^{2}}, \\
A_{\mathrm{S}} & =a \rho_{e q}^{\prime} \frac{\varepsilon G_{E}^{\mathrm{p}} G_{E}^{\mathrm{s}}+\tau G_{M}^{\mathrm{p}} G_{M}^{\mathrm{s}}}{\varepsilon\left(G_{E}^{\mathrm{p}}\right)^{2}+\tau\left(G_{M}^{\mathrm{p}}\right)^{2}},
\end{aligned}
$$

where $a=-G_{\mu} q^{2} / 4 \pi \alpha \sqrt{2}$ and $\varepsilon=\left[1+2(1+\tau) \tan ^{2}(\theta / 2)\right]^{-1}\left(G_{\mu}\right.$ is the Fermi constant from the muon decay, $\alpha$ the fine structure constant and $\theta$ the scattering angle in the laboratory frame). Electroweak radiative corrections evaluated within the $\overline{\mathrm{MS}}$ renormalisation scheme are contained in $\rho_{e q}^{\prime}$ and $\hat{\kappa}_{e q}^{\prime}$, while $\hat{s}_{Z}^{2}$ is the sine squared of the weak mixing angle at the $Z$-boson mass [5]. The electromagnetic form factors are measured and $G_{A}$ can be calculated using the weak proton charge from $\beta$ decay measurements and a dipole form for the $q^{2}$ dependence. Radiative corrections of the axial proton coupling are included in the value of $G_{A}$ ([6] and references therein). Measuring $A^{\mathrm{PV}}$ and subtracting $A_{0}$ gives a linear combination of the electric and magnetic strangeness form factors $G_{E}^{s}$ and $G_{M}^{s}$. These in turn can be separated through a Rosenbluth-type method by performing the measurement at the same $q^{2}$ but different scattering angles.

Four collaborations have performed measurements of the strangeness vector form factors: SAMPLE at MIT-BATES, Happex and G0 at TJNAF and A4 at MAMI. A survey of the data from these experiments is shown in fig. 1 . At $\left|q^{2}\right|$ of about $0.1(\mathrm{GeV} / c)^{2}$ the data set is fairly complete. The separation of $G_{M}^{s}$ and $G_{E}^{s}$ is possible and also a direct measurement of $G_{A}$ in agreement with theoretical estimations is available. 


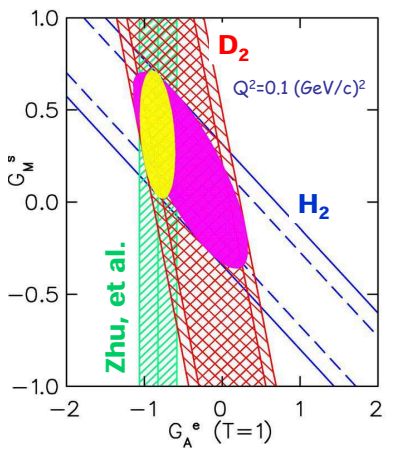

(a)

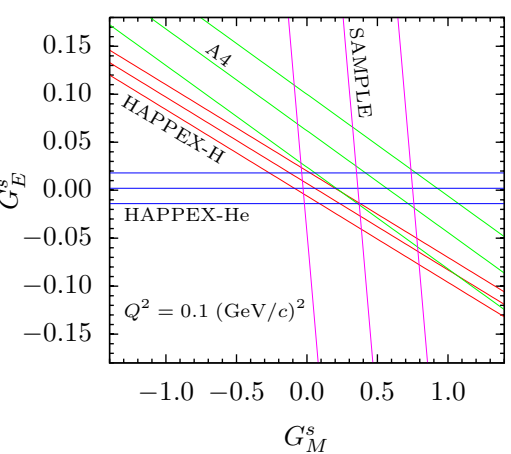

(b)

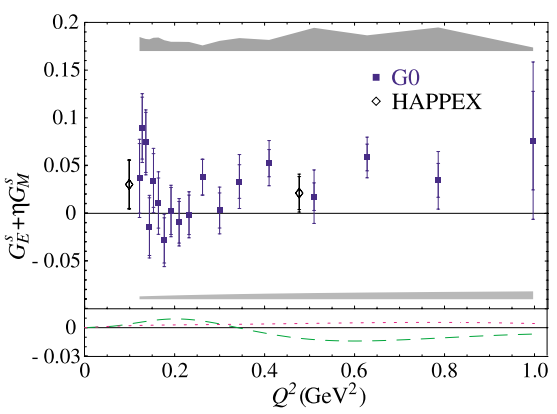

(c)

Figure 1: (a) SAMPLE backward angle measurements on $\mathrm{H}_{2}$ and $\mathrm{D}_{2}$ [8]. The purple ellipse is the combination of the two measurements, the yellow one results from the $\mathrm{H}_{2}$ measurement together with the $G_{A}$ estimation of [6]. (b) Available data at $0.1(\mathrm{GeV} / c)^{2}$ come from the Happex collaboration [9] (forward, $\mathrm{H}_{2}$ and $\mathrm{He}$ targets), from A4 [10] (forward, $\mathrm{H}_{2}$ ) and SAMPLE [8] (backward, $\mathrm{H}_{2}$ ). The SAMPLE band is calculated using $G_{A}$ from [6]. (c) Results of the $\mathrm{G} 0$ experiment with a $\mathrm{H}_{2}$ target [11]. The setup was designed for measuring simultaneously at different $q^{2}$ values. The Happex points at $0.1(\mathrm{GeV} / c)^{2}[9]$ and $0.477(\mathrm{GeV} / c)^{2}[12]$ are also shown.

For measuring the helicity asymmetry, a longitudinally polarised electron beam is scattered on a hydrogen, deuterium or helium target and the rate of (quasi-)elastic scattering events is measured for each helicity state. In the case of the A4 experiment single events are counted and the number $N_{R, L}$ of detected events is used for calculating the asymmetry $A^{\mathrm{PV}}=\left(N_{R}-N_{L}\right) /\left(N_{R}+N_{L}\right)$.

The order of magnitude of $A^{\mathrm{PV}}$ is fixed by the ratio $q^{2} / M_{Z}^{2}$. At $\left|q^{2}\right| \sim 0.1(\mathrm{GeV} / c)^{2}$ one has $A^{\mathrm{PV}} \leq 10^{-5}$. The smallness of this quantity strictly dictates the experimental design. For a counting experiment the statistical error of the asymmetry is $\delta A_{\text {stat }}=1 / \sqrt{N}$ (with $N=N_{R}+N_{L}$ ). Willing to achieve $\delta A_{\text {stat }} \sim 10^{-7}$ one needs to detect $N \sim 10^{14}$ events. Assuming a beam time of e.g. 1000 hours, this requires the capability of registering events at a rate of $10 \mathrm{MHz}$. A setup with high luminosity, large angle acceptance and a suitably fast detector has to be devised.

On the systematics side, even very small helicity correlated fluctuations of the beam parameters can bias the measurement. Such fluctuations have to be kept low, monitored, and their impact on the measured asymmetry has to be carefully estimated. A last crucial topic is the measurement of the degree of polarisation of the beam. Its uncertainty dominates the systematic error in the case of the A4 experiment, although it can not be discussed here.

A review of the experimental solutions for these measurements can be found in [7].

\section{The A4 experiment at Mainz}

The A4 experiment takes place at the MAMI accelerator facility at Mainz. The MAMI polarised electron beam is struck on a liquid hydrogen or deuterium target and the scattered particles are detected by an electromagnetic calorimeter, placed either at forward or backward scattering angles. The beam polarisation is measured and monitored with four different polarimeters.

Polarised electrons are obtained by irradiating a super-lattice GaAs photocathode with circularly polarised laser light. The achieved polarisation is usually between $70 \%$ and $80 \%$ with a beam intensity of $20 \mu \mathrm{A}$. A Pockels cell is used for switching the polarisation sign every $20 \mathrm{~ms}$ and 

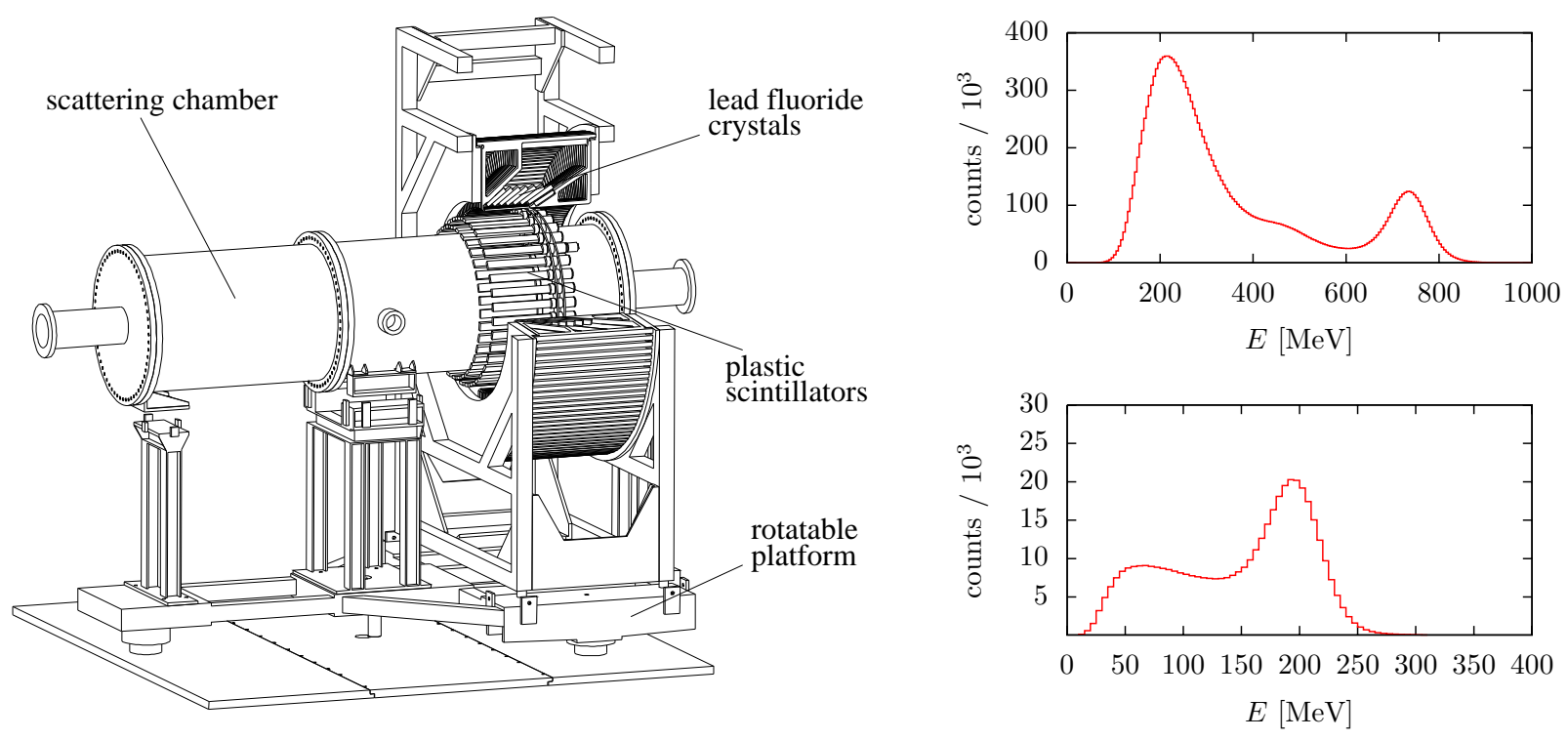

Figure 2: Left: Technical drawing of the A4 detector setup. Right: Energy spectra measured at forward (top) and backward (bottom) angles.

on the same time basis the beam parameters are measured with beam monitors placed at different locations along the beam line. A $\lambda / 2$ waveplate can be put into or out of the laser beam, changing the sign of the polarisation. This offers a systematic check of the measured asymmetry which has to change in sign but not in magnitude by moving the waveplate. The electrons are extracted by the source and injected by a $4 \mathrm{MeV}$ linac into the accelerator. This consists of three race track microtrons in cascade and a double sided microtron, giving a maximum energy of $1.5 \mathrm{GeV}$ and working in continuous wave [15].

An absolute measurement of the beam polarisation is performed once a week with a Møller polarimeter and online with a Compton backscatter polarimeter. A Mott polarimeter before the injector is calibrated using the Møller measurement and gives a polarisation value every two days. The interpolation between these measurements is made possible by a transmission Compton polarimeter placed before the beam dump.

The liquid hydrogen target is kept at $14 \mathrm{~K}$ by a high power helium refrigeration system, absorbing $100 \mathrm{~W}$ of heat at a beam current of $20 \mu \mathrm{A}$. The temperature fluctuations are smaller than $10^{-3}$. The target cell is $10 \mathrm{~cm}(23.4 \mathrm{~cm})$ long for the forward (backward) measurement. Fluctuations of the target density are monitored by 8 water Cherenkov detectors integrating the flux of particles scattered between $4^{\circ}$ and $10^{\circ}$ of polar angle and over the whole azimuthal angle range.

The detector system of the A4 experiment is shown in fig. 2. The particle detection happens in a fully absorbing electromagnetic calorimeter made of $1022 \mathrm{PbF}_{2}$ crystals. This material is a pure Cherenkov radiator and thus intrinsically fast, allowing count rates up to $100 \mathrm{MHz}$. The energy resolution $(\Delta E / E \sim 4 \% / \sqrt{E / \mathrm{GeV}})$ is sufficient for separating elastic from inelastic events. The crystals are arranged in rings, symmetrically around the beam axis and covering a solid angle of $0.6 \mathrm{sr}$. The calorimeter is mounted on a rotatable platform for changing the setup between forward and backward angle measurements. The polar angle acceptance is $30^{\circ}$ to $40^{\circ}$ for the forward and 
Table 1: I. Helicity correlated differences $\Delta X_{i}$ in the mean value of the beam parameters and resulting instrumental asymmetries. II. Corrections applied to the raw asymmetry. Besides the beam polarisation ${ }^{1}$ and the instrumental asymmetries ${ }^{2}$, also the random coincidences between scintillator and calorimeter ${ }^{3}$, the pollution due to scattering off the aluminium nuclei of the target cell ${ }^{4}$ and the subtraction of the converted $\gamma$-background ${ }^{5}$ are taken into account.

\section{Instrumental asymmetries}

\begin{tabular}{lclc} 
Parameter & $X_{i}$ & & $a_{i} X_{i}[\mathrm{ppm}]$ \\
\hline Current asymmetry & -0.30 & $\mathrm{ppm}$ & -0.25 \\
Horiz. position diff. & -86.97 & $\mathrm{~nm}$ & +0.61 \\
Vert. position diff. & -23.84 & $\mathrm{~nm}$ & -0.86 \\
Horiz. angle diff. & -8.53 & $\mathrm{nrad}$ & -0.09 \\
Vert. angle diff. & -2.40 & $\mathrm{nrad}$ & +0.10 \\
Energy diff. & -0.41 & $\mathrm{eV}$ & +0.16
\end{tabular}

\section{Corrections}

\begin{tabular}{lcc} 
& Factor & Error \\
\hline \hline & 0.68 & 0.04 \\
\hline${ }^{1}$ Polarisation & Corr. $(\mathrm{ppm})$ & Error $(\mathrm{ppm})$ \\
\hline${ }^{2}$ Hel. corr. asym. & +0.14 & 0.39 \\
${ }^{3}$ Random coinc. & -0.19 & 0.02 \\
${ }^{4}$ Al windows & +0.29 & 0.04 \\
${ }^{5}$ Background subtr. & -1.49 & 0.28
\end{tabular}

$140^{\circ}$ to $150^{\circ}$ for the backward setup.

In the backward configuration there is a strong $\gamma$-background from the $\pi^{0}$ decay which can not be separated by the calorimetric energy measurement. For suppressing such a background the detector was equipped with a total of 72 plastic scintillators which distinguish between charged and neutral particles.

Example of energy spectra, measured with the calorimeter, are shown on the right in fig. 2. Every five minutes two such spectra, corresponding to each beam polarisation state, are obtained from each of the 1022 calorimeter modules. The peak of elastic scattering events is clearly visible and the number $N_{R, L}$ of these events can be extracted from it. These numbers are normalised to the mean target $\rho_{R, L}$ density measured during each polarisation state and from the resulting asymmetry $A^{\text {meas }}$ the parity-violating asymmetry $A^{\mathrm{PV}}$ is extracted according to

$$
A^{\text {meas }}=\frac{N_{R} / \rho_{R}-N_{L} / \rho_{L}}{N_{R} / \rho_{R}+N_{L} / \rho_{L}}=P A^{\mathrm{PV}}+\sum_{i} a_{i} \Delta X_{i}
$$

where $P$ is the beam polarisation and the terms in the sum are instrumental asymmetries due to systematic shifts $\Delta X_{i}$ of some beam parameter $X_{i}$ between the two polarisation states (see table 1). These $\Delta X_{i}$ are measured and the coefficients $a_{i}$ are determined through a multilinear regression.

\section{Latest results}

The backward angle measurement at $-q^{2}=0.22(\mathrm{GeV} / c)^{2}$ is summarised in table 1 , where the applied corrections and a budget of the systematic errors is given. In fig. 3 the systematic check of inverting the beam polarisation at the source by a $\lambda / 2$ waveplate is shown. The final result for the parity-violating asymmetry is

$$
A^{\mathrm{PV}}=\left(-17.23 \pm 0.82_{\text {stat }} \pm 0.89_{\text {syst }}\right) \text { ppm. }
$$

The expected asymmetry setting the strange vector form factors equal to zero is $A_{0}=(-15.87 \pm$ 1.22) ppm, being the error due to the uncertainty of the other form factors involved. The resulting 


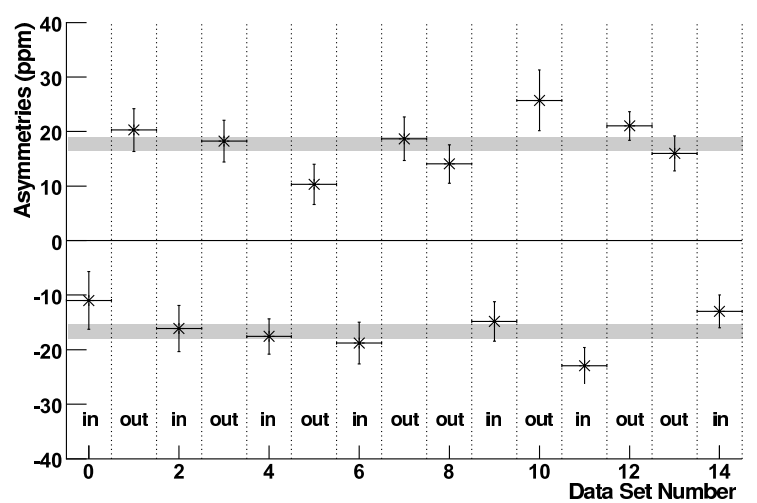

Figure 3: Result of the systematic check of inverting the polarisation by a $\lambda / 2$ wave plate.

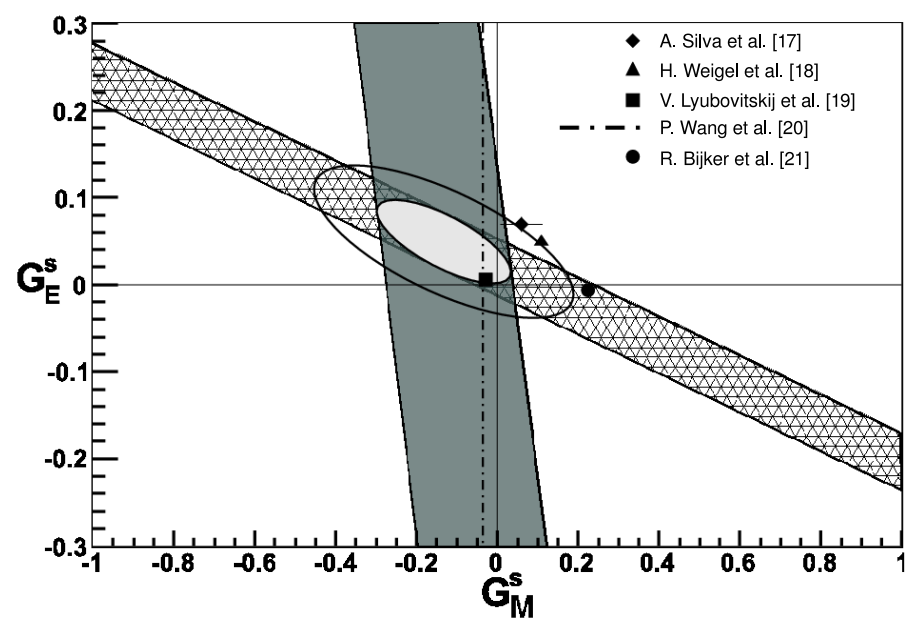

Figure 4: A4 forward and backward angle results at $0.22(\mathrm{GeV} / c)^{2}[13,14]$. The bands, like the filled ellipse, show to the one-standard-deviation uncertainty. The empty ellipse corresponds to two standard deviations.

linear combination of $G_{E}^{s}$ and $G_{M}^{s}$ is $G_{M}^{s}+0.26 G_{E}^{s}=-0.12 \pm 0.11_{\exp } \pm 0.11_{\mathrm{FF}}$ [14], where the last error is the theoretical error due to the other form factors. Combining it with the forward A4 result [13] $G_{E}^{s}+0.224 G_{M}^{s}=-0.020 \pm 0.029_{\exp } \pm 0.016_{\mathrm{FF}}$, on can separate the two strangeness form factors: $G_{M}^{s}=-0.14 \pm 0.11_{\exp } \pm 0.11_{\mathrm{FF}}$ and $G_{E}^{s}=-0.050 \pm 0.038_{\exp } \pm 0.019_{\mathrm{FF}}$. In fig. 4 the two A4 measurements at this $q^{2}$ are shown.

\section{Conclusions}

A number of parity-violating electron scattering measurements have been carried out successfully at three different facilities. The helicity asymmetries have been measured with the planned accuracy and the strangeness vector form factors have been extracted. They are, at least at low $q^{2}$ values, compatible with zero. This rules out a broad variety of theoretical approaches and model calculations giving large values for these quantities.

The experimental programme is still ongoing and further data are upcoming. Backward angle data at $0.22(\mathrm{GeV} / c)^{2}$ on $\mathrm{H}_{2}$ from the $\mathrm{G} 0$ Collaboration as well as on $\mathrm{D}_{2}$ from $\mathrm{A} 4$ are being 
analysed and will settle the situation at this $q^{2}$. Two forward measurements on $\mathrm{H}_{2}$ at larger $q^{2}$ $\left(\sim 0.6(\mathrm{GeV} / c)^{2}\right)$ are being performed by the Happex and A4 collaborations.

\section{References}

[1] A. Manohar, H. Georgi, Nucl. Phys. B 234 (1984), 189-212

[2] D.B. Kaplan, A. Manohar, Nucl. Phys. B 310 (1988), 527-547

[3] B. Kubis, R. Lewis, Phys. Rev. C 74 (2006), 015204

[4] M.J. Musolf et al., Phys. Rep. 239 (1994), 1-178

[5] C. Amsler et al., Phys. Lett. B 667 (2008), 1

[6] S.L. Zhu et al., Phys. Rev. D 62 (2000), 033008

[7] K.S. Kumar, P.A. Souder, Prog. Part. Nucl. Phys. 45 (2000), 333-395

[8] D. T. Spayde et al., Phys. Lett. B 583 (2004), 79-86. T.M. Ito et al., Phys. Rev. Lett. 92 (2004), 102003

[9] A. Acha et al. (Happex Collaboration), Phys. Rev. Lett. 98 (2007), 032301

[10] F.E. Maaset al., Phys. Rev. Lett. 94 (2005), 152001

[11] D.S. Armstrong et al. (G0 Collaboration), Phys. Rev. Lett. 95 (2005), 092001

[12] K.A. Aniol et al. (Happex Collaboration), Phys. Rev. C 69 (2004), 065501

[13] F.E. Maas, et al., Phys. Rev. Lett. 93 (2004), 022002

[14] S. Baunack et al., Phys. Rev. Lett. 102 (2009), 151803

[15] See contribution of H. Merkel to this conference for details about the MAMI facility.

[16] A. Silva, H. C. Kim, D. Urbano, and K. Goeke, Phys. Rev. D 74 (2006), 054011

[17] H. Weigel et al., Phys. Lett. B 353 (1995), 20

[18] V. E. Lyubovitskij, P. Wang, T. Gutsche, and A. Faessler, Phys. Rev. C 66 (2002), 055204

[19] P. Wang, D. B. Leinweber, A. W. Thomas, and R. D. Young, Phys. Rev. C 79 (2009), 065202

[20] R. Bijker, J. Phys. G 32 (2006), L49 